\title{
Conic Asset Pricing and The Costs of Price Fluctuations.
}

\author{
Dilip B. Madan \\ Wim Schoutens \\ Robert H. Smith School of Business \\ University of Maryland \\ K. U. Leuven \\ Leuven, Belgium
}

College Park, MD. 20742

February 20, 2017 


\begin{abstract}
Prudent upper and lower valuations from the literature on arbitrage free two price economies provide risk characteristics driving required returns. The risk characteristics assess the risk of price fluctuations. The difference between the upper and lower prudent valuations can be viewed as a capital charge. In addition the lower valuation assesses the down side tail risk. The required risk characteristics may be estimated on a daily basis from past data and we elaborate on how to perform such upper and lower valuations using distorted expectations. Details are provided for calculations using just the raw data, or by first fitting a probability distribution, or in terms of estimated arrival rates for jumps. The valuations are obtained with a dynamic calibration of a parameteric distortion on the S\&P 500 index options market. Results for required returns based on capital charges and down side risk compensation show an improvement when risk is represented by the arrival rates of jump sizes. For risk assessments based on arrival rates, capital charges constitute between $67 \%$ to $85 \%$ of the required return. The rest being a charge for downside risk exposures. After the introduction of risk characteristics into required returns there is little scope for covariation measures like asset betas. Different proposed constructions for required returns deliver differences in the value of an invested dollar and associated differences in asset rankings across time.

Keywords: Probability Distortion, Measure Distortion, Self Decomposable Law, Variance Gamma Model.
\end{abstract}

JEL Codes: G10, G12, G13.

\title{
1 Introduction
}

Two risk characteristics are proposed as drivers of risk compensations embedded in required returns. The required risk characteristics may be estimated on a daily basis from past data. The risk characteristics are based on prudent upper and lower valuations as they appear in abstract models of arbitrage free two price economies (Jouini and Kallal (1995), Bion Nadal (2009), Guasoni, Lepinette and Rasonyi (2012), Madan (2015a)). In the abstract model the law of one price is replaced by two prices, one, lower and the other, higher, evaluating how far up and down prices may reasonably be viewed as fluctuating. Recognizing that prices must continuously be changing to support the price with a return the focus of risk turns from the explanation of prices to the nature of their inherent and necessary fluctuation.

Required returns traditionally are looked at as proportional to a single beta. The law of one price coupled with the absence of no arbitrage leads to linear valuation principles (Ross (1978)). The result is a covariation based explanation for excess returns (Back 2010)). The beta is an example of such a covariation based measure of risk exposure originally arising in the capital asset pricing models of Sharpe (1964), Lintner (1965) and Mossin (1966). Multi factor extensions followed on from Ross (1976). The literature since (Brennan, Chordia and Subrahmanyam (1998), Chordia, Goyal and Shanken (2015)) has consid- 
ered the addition of firm characteristics as additional explanatory factors that are available at best at a monthly frequency. Firm characteristics were recast as a form of betas by Fama and French $(1992,1993)$. Here an alternative risk characteristics approach to required returns is developed.

In a two price context, one expects risks with considerable down side tail exposure to have a smaller lower price. Similarly risks with a significant up side tail exposures lead to a larger higher price. The spread between the higher and lower price was taken as a capital charge in Carr, Madan and Vicente Alvarez (2011). The argument being that such capital amounts allow for both entry and exit from a trade on adverse terms in both directions. In addition to this capital spread the lower price may be viewed as a further measure of downside risk. The two risk characteristics considered in the paper are (i) the higher lower price spread and (ii) the lower price. Results are reported using these two new risk characteristics, both with and without the addition of a beta. It is useful to note up front that the units of the two proposed risk characteristics are identical with those of the dependent variable, the expected cash flow to a dollar. They are all measured in dollars.

Implementation of this alternative risk characteristics approach to required returns employs specific computations for the higher and lower prices theoretically prevailing in underlying two price economies. In the context of single or multiperiod models this has been accomplished using probability distortions, see for example Madan and Schoutens (2016) and the references cited therein. The analysis can then proceed with all computations at the level of daily data. The paper reports the results for required returns using daily data with no modelling assumptions about returns.

However, critical to the analysis is the estimation of expected returns. The difficulty with estimating expected returns has long been noted. Black (1993) pointed out that estimation by averaging will often require very long data records and argued that theory could help circumvent data deficiencies. Madan (2017) shows how using knowledge about the full uncentered distribution improves the efficiency of expected return estimation beyond averaging.

The presumed knowledge of the full return distribution being leveraged in the estimation is the recognition that prices must change to support the price with a return. Furthermore the price changes must be surprises therefore occuring at surprise times. This makes the price process a pure jump process. But given the large number of changes or shocks in a day it is suggested that one considers a pure jump limit law. As a consequence the underlying pure jump process is not only infinitely divisible but must by virtue of being a limit law, also be self decomposable (Lévy (1937), Khintchine (1938)).

Such self decomposable pure jump laws have a special structure to their jump arrival rates (Sato (1999)). The arrival rates scaled by the absolute jump size must be decreasing functions of the jump size. A particularly simple, tractable and empirically robust and adequate example is the variance gamma law (Madan and Seneta (1990), Madan Carr and Chang (1998)). The paper reports results on employing a variance gamma full distribution for all required computations. This includes the mean returns and the distortion of variance gamma tail prob- 
abilities for the higher and lower prudent valuations.

A further issue with either a raw data analysis or the full distribution at a horizon is the selection of the horizon. Returns and their distributions only come into existence at some horizon, be it a minute, day, week, month or year. The longer horizons leave us short of data. A horizon as short as a day is a long period in markets. At the even shorter horizons, the entities of interest, like the probabilities of outcomes in specific intervals, converge to zero as the horizon gets shorter. The meaningful and estimable entities are then limits, as the horizon goes to zero, of the probabilities of interest divided by the horizon. A further advantage of working at the limit is that densities and distributions at any horizon can be complicated while the limiting objects given by the arrival rates of jumps as a function of the jump size are much simpler analytical objects. The paper presents results at the level of arrival rate functions for all required computations. This includes the exponential variation as the reward function and measure distorted variations replacing probability distortions for the higher and lower prudent valuations.

The probability distortions employed on data and variance gamma distributions are given parametrically as are the measure distortions. The distortion parameters must be calibrated from market data. It may be observed that if distortion parameters are not calibrated daily but frozen for longer periods then results can be adversely impacted on account of delayed or obsolete risk assessments. From the options market one may observe risk neutral probabilities and risk neutral arrival rate functions. Their physical counterparts may be estimated from time series data on the relevant underlying asset, extended when necessary to the option maturity. The distortions to be applied to the physical tail probabilities or measures must be sufficient to dominate in specific ways the risk neutral counterparts with a view to organizing higher and lower valuations that contain risk neutral prices between the two valuations. The distortion parameters are calibrated daily from option data on S\&P 500 index options on the day and time series date for a year preceeding the day to organize the required domination.

The results demonstrate considerable stability delivered by employing the analytical structure of applying measure distortions to the limiting arrival rate functions as compared with the use of probability distortions applied either to probability models or the empirical distribution function. The two risk characteristics are both highly significant in explaining expected returns especially when applying measure distortions. There is also little room or reason for the inclusion of betas once risk characteristics have been introduced. Capital charges constitute between 67 to 85 percent of required return based on measure distortions. The rest may be attributed to downside risk.

Additional to the introduction of risk characteristics in formulating required returns we report on the value of an invested dollar as an investment objective. When a dollar is invested in a risky enterprise it gains access to risky cash flows with some hopefully positive expected cash flow. Given the presence of risk there is an anticipated required return over a fixed time horizon. If the expected cash flow on a dollar equals the required return then the fair value 
of the dollar is unity. The value may be above or below unity depending on whether the expected cash flow on the dollar is above or below the required return. Hence, two entities determine the value of the invested dollar: (i) the expected cash flow on the dollar, and (ii) the required return on the dollar. The value of the invested dollar is then the ratio of these two entities.

A widely used investment objective is the generation of alpha. The alpha of an investment is the excess of the expected cash flow over the required return. However, from the perspective of the value of invested dollars pure alpha seeking strategies can be misdirected. This is because the value of the invested dollar only rises on increasing alpha per unit of required return. High alphas attained with unusually high required returns may lower the value of the invested dollar.

The value of the invested dollar is thus alpha per unit beta or the alpha beta ratio. Performance rankings based on these alpha beta ratios can be different than those based just on alpha. Comparisons of these for some 229 stocks, 9 sector exchange traded funds (ETF's) and the S\&P 500 index (SPX) are reported on in the paper. The different constructions for the value of an invested dollar deliver different asset rankings across time.

The outline of the rest of the paper is as follows. Section 2 introduces the uncentered variance gamma model, its density, arrival rate function and estimation procedures from uncentered daily data. Section 3 introduces the lower and higher prudent valuations using probability distortions to be applied to the raw data or the estimated variance gamma distribution at the daily horizon. Section 4 introduces higher and lower prudent valuations based on measure distortions of arrival rate functions. The calibration of both probability and measure distortions parameters to be used on any particular day are presented in Section 5. Section 6 reports the results on required returns. Section 7 presents a comparison of classical alpha rankings with their alpha beta counterparts along with a risk characteristics approach to the value of an invested dollar and associated asset rankings. Section 8 concludes.

\section{Distributional Modeling for Financial Returns}

It has been a common practice in modeling return distributions to first estimate the mean by taking a sample average and to then estimate a centered model on the centered data. Such a procedure recognizes that random variables have a mean and a distribution about the mean, almost by definition. Hence a single modeling and estimation problem is split into two problems. The alternative would be to model the full distribution directly and to then estimate the uncentered model on the uncentered data.

Madan (2016) reports on the deterioration in the quality of fit to the data generated by first performing a centering of the data. The initial centering essentially introduces noise generated by a noisy estimate for the mean delivered by averaging. The performance of the estimation is considerably enhanced by directly estimating the uncentered model on the uncentered data. Madan (2017) goes on to demonstrate the increase in efficiency obtained in estimating the mean 
by directly modeling and estimating the full distribution directly.

Essentially direct averaging makes no use of how the random variable comes into existence and in the absence of leveraging such information one gets a poor estimate of the mean. As noted in the introduction Black (1993) warned against this practice and suggested that some theoretical analysis could assist the estimation. Madan (2017) develops such an analysis by recognizing some properties of the random variable under study.

The first of these properties is the necessary motion in asset prices and the nature of such motion. Abstracting from the relatively infrequent distribution of dividends, and recognizing that the assets must be held by someone, asset prices must change and on average rise to support the prices being paid with a return. Furthermore, the required changes cannot be predictable but must be surprises. As a consequence one accepts the hypothesis that the changes occur at unpredictable times. Technically these are Poisson arrival times for the moves of each possible jump size. As a consequence the price process or equivalently its logarithm is a pure jump process.

Additionally, the number of jumps involved being large, suggests a distribution of a limit law. The limit laws were studied and characterized by Lévy (1937) and Khintchine (1938) and are known as the self decomposable laws. Self decomposable random variables can be thought of as distributionally equivalent to a scaled down version of themselves plus an independent residual random variable. The pure jump self decomposable laws are infinitely divisible but with a further special structure for the arrival rate functions that announce the Poisson arrival rates of jumps as functions of the jump size. This special structure is described in Sato (1999) and requires that the arrival rate scaled by the absolute value of the jump size be a decreasing function of the absolute jump size.

A particularly simple, tractable and empirically adequate example of such a law is the variance gamma law (Madan and Seneta (1990), Madan, Carr and Chang (1998)). The scaled arrival rate function is just a negative exponential of the absolute jump size and clearly decreasing. This model is employed here as the default distributional model for daily returns. Its arrival rate function is employed for analysis in the continuous time limit arising on letting the time horizon tend to zero. The details of the variance gamma model follow.

\subsection{Variance Gamma Details}

The variance gamma model is the distribution at unit time of the variance gamma process. The variance gamma process is given by Brownian motion with drift $\theta$, volatility $\sigma$ that is time changed by a gamma process with unit

mean rate and variance rate $\nu$. Let $X$ be the variance gamma random variable then the characteristic function of $X$ is (Madan, Carr and Chang (1998))

$$
E[\exp (i u X)]=\left(\frac{1}{1-i u \theta \nu+\sigma^{2} \nu u^{2} / 2}\right)^{\frac{1}{\nu}} .
$$


The random variable $X$ can be thought as generated according to

$$
X=\theta U+\sigma \sqrt{U} Z,
$$

where $U$ is gamma distributed with shape and scale parameters equal to $1 / \nu$ and $Z$ is a standard normal variate. This representation can be used to simulate variance gamma random variables from gamma and standard normal variates.

Carr, Geman, Madan and Yor (2002) developed the arrival rate function for the variance gamma in the form

$$
k(z)=C \frac{\exp (-M z)}{z} ; k(-z)=C \frac{\exp (-G z)}{z}, z>0 .
$$

The mapping from the $C, G$ and $M$ parameters to $\sigma, \nu$ and $\theta$ is

$$
\nu=\frac{1}{C}, \theta=C\left(\frac{1}{M}-\frac{1}{G}\right), \sigma=\sqrt{\frac{2 C}{G M}} .
$$

From the arrival rate function it is observed that the variance gamma random variable is a self decomposable random variable with a simple negative exponential structure for the functions $z k(z), z k(-z)$ for $z>0$. The variance gamma process is one of infinite activity as the arrival rate function integrates to infinity on both sides of zero. However, it is a process of finite variation as the integral of jump sizes $z$ against the arrival rate function $k(z)$ is finite on both sides of zero. The model provides parametric control of skewness via $\theta$ and kurtosis via $\nu$.

The density has a closed form (Carr and Madan (2014)) given by

$f_{C G M}(x)=\frac{(G M)^{C}}{2^{C-1} \Gamma(C) \sqrt{2 \pi}\left(\frac{G+M}{2}\right)^{C-1 / 2}} \exp \left(\frac{G-M}{2} x\right)|x|^{C-1 / 2} K_{C-1 / 2}\left(\frac{G+M}{2}|x|\right)$

where $K_{\nu}(x)$ is the modified Bessel function. This density is used to evaluate digital tail probabilities that are matched by least squares to empirical tail probabilities as described in more detail in the next section.

The expected rate of return per unit time then is

$$
\begin{aligned}
E\left[\left(e^{X}-1\right)\right] & =E[R]=\left(\frac{1}{1-\theta \nu-\frac{\sigma^{2} \nu}{2}}\right)^{\frac{1}{\nu}}-1 \\
& =\left(\frac{G M}{(G+1)(M-1)}\right)^{C}-1
\end{aligned}
$$

Further the continuously compounded rate of return $X=\ln (1+R)$ has expectation $\theta$ or the equivalent $(C, G, M)$ expression in 1 .

\subsection{Estimation based on tail probabilities}

Many estimation methods including maximum likelihood amount to matching theoretical to empirical moments. Madan (2015) argues that if the data generating model is not clear, the use of bounded moments, like tail probabilities, 
are expected to perform better than maximum likelihood as they are not that affected by outliers that can adversely impact unbounded moments. There are other bounded moments like those for trignometric functions. However, tail probabilities have a convenient structure for unimodal models (like self decomposable laws): probabilities decrease with distance from the mode.

We estimate variance gamma parameters in the original $\sigma, \nu$ and $\theta$ parameterizations by matching selected lower and upper tail probabilities. Given uncentered log price relatives $x_{1}, x_{2}, \cdots, x_{T}$ construct the empirical distribution function by ranking the $x_{t}: x_{(1)}<x_{(2)<}, \cdots<x_{(T)}$. The empirical distribution function is then $t / T$ at $x_{(t)}$. For probability levels ranging from one percent to 99 percent we determine by interpolation the point $y_{i}$ corresponding to probability $i / 100, i=1,2, \cdots, 99$. For negative $y_{i}$ assign the lower tail probability of $\pi_{i}=i / 100$ while positive $y_{i}$ are assigned the upper tail probability $\pi_{i}=1-i / 100$.

The theoretical distribution function $\widehat{F}_{i}$ at $y_{i}$ is

$$
\widehat{F}_{i}=\sum_{j \leq i} p_{j}\left(y_{j}-y_{j-1}\right)
$$

where the $p_{i}$ are the variance gamma density (2) evaluated at $y_{i}$ given the parameters and $y_{1}-y_{0}=y_{2}-y_{1}$. The three unknown parameters are estimated on minimizing

$$
\sum_{i}\left(\frac{\pi_{i}-\widehat{\pi}_{i}}{\pi_{i}}\right)^{2}, \widehat{\pi}_{i}=\left\{\begin{array}{cc}
\widehat{F}_{i} & y_{i}<0 \\
1-\widehat{F}_{i} & y_{i}>0
\end{array}\right.
$$

Note the minimization is in terms of percentage errors so as to give a reasonable weight to the small tail probabilities.

\section{Lower and Upper Prudential Valuations using Probability Distortions}

Given the log price relative, $X$, for a horizon $h$ with return density $f(x, h)$, the expected cash flow to a dollar invested at the start of the period is given by

$$
E[R]=\int_{-\infty}^{\infty}\left(e^{x}-1\right) f(x, h) d x .
$$

Making the change of variable to $a=\left(e^{x}-1\right)$ and performing an integration by parts in $a$ we obtain that

$$
E[R]=-\int_{-\infty}^{0} d a \int_{e^{x}-1<a} f(x, h) d x+\int_{0}^{\infty} d a \int_{e^{x}-1>a} f(x, h) d x .
$$

Denote the physical probabilities by $P$ and define

$$
P(A)=\int_{A} f(x, h) d x
$$


In terms of $P$ we may write

$$
E[R]=-\int_{0}^{\infty} P\left(R^{-}>x\right) d x+\int_{0}^{\infty} P\left(R^{+}>x\right) d x .
$$

Expression (5) is a well known Choquet (1953) expectation computation applied here to the variable $R$.

Lower prudential valuations based on probability distortions attain conservative valuations by employing a concave distribution function $\Psi(u) \geq u$, $0 \leq u \leq 1$. The distortions lift the probabilities of the loss events on the left while simultaneously employing the convex distortion

$$
\widehat{\Psi}(u)=1-\Psi(1-u) \leq u,
$$

to lower the gain probabilities on the right.

The lower prudential valuation based on probability distortions is then

$$
\left.\left.L=-\int_{0}^{\infty} \Psi\left(P\left(\left(e^{X}-1\right)^{-}>a\right)\right)\right) d a+\int_{0}^{\infty} \widehat{\Psi}\left(P\left(\left(e^{X}-1\right)^{+}>a\right)\right)\right) d a .
$$

The upper prudential valuation applied to modeling liability add-ons operate the other way, lifting the right tail and lowering the left tail to obtain

$$
\left.\left.U=-\int_{0}^{\infty} \widehat{\Psi}\left(P\left(\left(e^{X}-1\right)^{-}>a\right)\right)\right) d a+\int_{0}^{\infty} \Psi\left(P\left(\left(e^{X}-1\right)^{+}>a\right)\right)\right) d a .
$$

The greater the concavity of $\Psi$ or the convexity of $\widehat{\Psi}$ the smaller is the lower valuation and the larger is the higher valuation. A single parameter distortion termed minmaxvar was introduced in Cherny and Madan (2009) and is given by

$$
\Psi^{(\gamma)}(u)=1-\left(1-u^{\frac{1}{1+\gamma}}\right)^{1+\gamma}, \gamma>0,
$$

where the parameter $\gamma$ controls the concavity or stress level of the distortion.

\subsection{Prudential Valuations and Covariations}

The lower and upper valuations (Madan and Schoutens (2016)) may be seen to be infima and suprema of expectations under alternative test measures $J \in \mathcal{M}$. In this case it may be observed that

$$
\begin{aligned}
L & =\inf _{J \in \mathcal{M}} E^{J}\left[\left(e^{X}-1\right)\right] \\
U & =\sup _{J \in \mathcal{M}} E^{J}\left[\left(e^{X}-1\right)\right] .
\end{aligned}
$$

The lower and upper valuations are then also based on infima and suprema of numerous covariations as opposed to a single beta for it is also the case that

$$
\begin{aligned}
L & =\inf _{J \in \mathcal{M}} E^{P}\left[\frac{d J}{d P}\left(e^{X}-1\right)\right] \\
U & =\sup _{J \in \mathcal{M}} E^{P}\left[\frac{d J}{d P}\left(e^{X}-1\right)\right],
\end{aligned}
$$


where $E^{J}$ denoted the expectation taken under the measure $J$.

The set of alternative measures $\mathcal{M}$ in this case (Madan and Schoutens (2016)) is here given by all probabilities $J$ with the property

$$
J(A) \leq \Psi(P(A)), \text { for all } A .
$$

Hence the greater the stress level or concavity of $\Psi$ the wider the class of alternative measures and the smaller or larger are the lower or the upper valuations.

Given a data set of returns over the past year, for example, one may evaluate using the minmaxvar distortion $\Psi^{(\gamma)}$ for a selected level of $\gamma$ the lower, $L$, and upper, $U$, valuations by integrating the distorted empirical tail probabilities in the expressions (6) and (7). If one has estimated the variance gamma model instead on the daily data, one may instead integrate distorted model tail probabilities.

The capital charge based on being able to trade in both directions on adverse terms is then $U-L$ following Carr, Madan and Vicente Alvarez (2011). The down side risk exposure is $L$. In this way one may evaluate for each asset $i$, on each day $t$, the capital charges $\left(U_{i t}-L_{i t}\right)$ and down side risk exposure $L_{i t}$ as two risk characteristics that are in the same units as the cash flows themselves.

The risk characteristics reflect market concerns about fluctuating prices. Essentially how far up and down can they be safely considered to be able to go and what is the status of down side tail risks. The capital charge and the lower price respectively address these issues via appropriate selection of stress levels of distortions.

In a classical one price economy the focus is on explaining the price as a covariation with a model for a single measure change seen as an aggregate factor beta exposure. Given that the price is by necessity in a state of continuous change we give up on such an agenda. Instead we focus attention on reasonable measures for the risk characteristics and then ask whether expected returns build in charges for these risk characteristics instead. For completeness we shall investigate the use of a traditional beta as well.

\subsection{Illustrative Computation of Lower and Upper Pruden- tial Valuations}

This subsection illustrates the computation of expectations and distorted expectations using the Choquet formulations for both. The variance gamma model was fit to the S\&P 500 return data for 252 days prior to September 28, 2015. The estimated parameter values for $\sigma, \nu$ were respectively $0.0095,0.8552$ and $\theta$ was 7.947 basis points. Figure 1 presents a graph of the tail probabilities observed in the data along with the fitted model tail probabilities. Also presented are the distorted tail probabilities as they are distorted for the lower and upper prudential valuation calculations for the stress level of 0.15 for the distortion minmaxvar. Using model probabilities, the expectation is the difference between the areas under the red curve, above and below zero. Similarly the lower and upper prudential valuations are the corresponding differences of areas under 


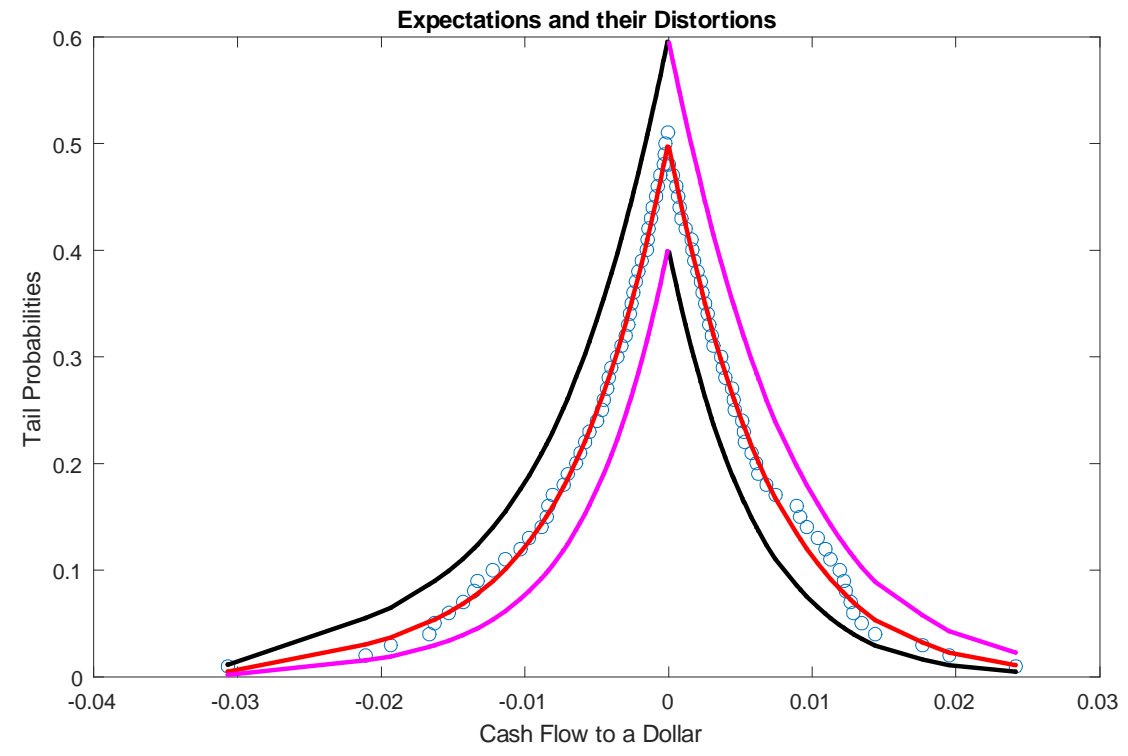

Figure 1: Tail probabilities as observed in data are shown by circles. The model probabilities are in red while the lower and upper distorted probabilities are in black and magenta respectively.

the black and magenta curves respectively. The three values, lower, expectation and upper are respectively in basis points $-33.15,-7.50$ and 16.78. Clearly a critical issue is the choice of the stress level to be used for the lower and upper valuations and this is addressed in Section 6 as noted in the introduction.

\section{Variations and Measure Distorted Lower and Upper Variations}

In the limit as the horizon goes to zero and the relevant probabilities are deflated by the horizon, the relevant magnitudes converge to the arrival rate function that for self decomposable limit laws is no longer a density. For all self decomposable or limit laws the arrival rate function integrates to infinity. The aggregate arrival rates are then infinite and the associated processes possess infinitely many jumps in any interval. One can then no longer speak of expectations or moments and integrals of functions of the jump size against the arrival rate function are called variations. Of particular interest is the exponential variation.

Suppose that the price process $S(t)$ has a logarithm $X(t)$ that is a pure jump process. We refer to Barndorff-Nielsen and Shiryaev (2010, page 50) for greater 
details. By construction

$$
S(t)=S(0) \exp (X(t))
$$

and the continuously compounded return to time $h$, defined by $\mu(h)$ is

$$
S(0) \exp (\mu(h))=E[S(h)] .
$$

Denote by $k(x, t)$ the arrival rate of a jump of size $x$ at time $t$ for the pure jump process $X(t)$. An expression for the characteristic function of $X(t)$ in terms of the arrival rate functions evaluated at $-i$ yields

$$
E[\exp (X(t))]=\exp \left(\int_{0}^{t} \int_{-\infty}^{\infty}\left(e^{x}-1\right) k(x, s) d x d s\right)
$$

and equivalently

$$
\mu(h)=\int_{0}^{h} \int_{-\infty}^{\infty}\left(e^{x}-1\right) k(x, s) d x d s .
$$

Taking the derivative with respect to $h$ evaluated at $h=0$ we get that

$$
\mu^{\prime}(0)=\int_{-\infty}^{\infty}\left(e^{x}-1\right) k(x) d x,
$$

where we now denote $k(x, 0)$ by $k(x)$. Hence it is the case that the exponential variation is

$$
\int_{-\infty}^{\infty}\left(e^{x}-1\right) k(x) d x=\lim _{h \downarrow 0} \frac{\mu(h)}{h} .
$$

This motivates the use of the exponential variation as the continuous time concept for reward measured strictly in terms of the arrival rate function.

The reward in the continuous limit is the exponential variation of the cash flow to a dollar,

$$
V=\int_{-\infty}^{\infty}\left(e^{x}-1\right) k(x) d x .
$$

Applying integration by parts one may write the variation as the integral of tail integrals, generalizing the Choquet type integrals of tail probabilities that were involved at fixed horizons with their densities and distribution functions. More specifically on making the change of variable $a=\left(e^{x}-1\right)$ and performing an integration by parts in $a$ one shows that

$$
\begin{aligned}
V & =-\int_{-\infty}^{0} \int_{\left(e^{x}-1\right)<a} k(x) d x d a+\int_{0}^{\infty} \int_{\left(e^{x}-1\right)>a}^{\infty} k(x) d x d a \\
& =-\int_{0}^{\infty} K\left(\left(e^{x}-1\right)^{-}>a\right) d a+\int_{0}^{\infty} K\left(\left(e^{x}-1\right)^{+}>a\right) d a,
\end{aligned}
$$

where the measure $K(A)=\int_{A} k(x) d x$. We next define lower and upper prudent variations by measure distortions. Consider first the prudent lower variation. 
The objective here is to get conservative by lifting the left tail measures and reducing the right tail measures. This is done by introducing two increasing functions $\Lambda^{+}$and $\Lambda^{-}$above and below the identity respectively, with the former concave and the latter convex to define the lower variation by

$$
V_{L}=-\int_{0}^{1} \Lambda^{+}\left(K\left(\left(e^{x}-1\right)^{-}>a\right)\right) d a+\int_{0}^{\infty} \Lambda^{-}\left(K\left(\left(e^{x}-1\right)^{+}>a\right)\right) d a .
$$

For the prudent upper variation the lift occurs on the right and the reduction on the left to get

$$
V_{U}=-\int_{0}^{1} \Lambda^{-}\left(K\left(\left(e^{x}-1\right)^{-}>a\right)\right) d a+\int_{0}^{\infty} \Lambda^{+}\left(K\left(\left(e^{x}-1\right)^{+}>a\right)\right) d a .
$$

One may define two increasing concave functions $\Gamma^{+}, \Gamma^{-}$and define

$$
\begin{aligned}
& \Lambda^{+}(x)=x+\Gamma^{+}(x) \\
& \Lambda^{-}(x)=x-\Gamma^{-}(x) .
\end{aligned}
$$

Note that $\Lambda^{-}(x) \leq x$ requires $\Gamma^{-}(x) \geq 0$.

Parameteric forms for the functions $\Gamma^{+}, \Gamma^{-}$were employed in Eberlein, Madan, Pistorius and Yor (2014) with

$$
\begin{aligned}
\Gamma^{+}(x) & =a\left(1-e^{-c x}\right)^{\frac{1}{1+\gamma}} \\
\Gamma^{-}(x) & =\frac{b}{c}\left(1-e^{-c x}\right) .
\end{aligned}
$$

These measure distortions are probability distortions applied to tail measures mapped into the unit interval using an exponential function of the tail measure and a fixed concavity coefficient of $c$. The derivative of $\Gamma^{-}$at zero is $b$. For symmetry of maximal risk charges applied to large tail measures one may take $a=b / c$. The final parameter is $\gamma$.

The lower and upper prudent variations can be expressed as infima and suprema over measures $L$ satisfying for all $A \subset \mathbb{R}-\{0\}$ that

$$
L(A) \in\left[\Lambda^{-}(K(A)), \Lambda^{+}(K(A))\right] .
$$

\subsection{Lower and Upper Prudential Variations by Measure Distortion of Variance Gamma Arrival Rates}

For variance gamma arrival rates for the log price relative we evaluate the lower and upper prudential valuations. The simple structure of the limiting arrival rate functions allows for analytical integration of the tail measures that are then to be distorted and integrated numerically.

The response $y$, on a dollar's holding of stock to a jump $x$ in the log price relative is

$$
y=\left(e^{x}-1\right)
$$


The arrival rates of jumps $y>-1$ are given by

$$
k_{Y}(y)=k_{X}(\ln (1+y)) \frac{1}{1+y}, y>-1 .
$$

The left tail for losses is given, with $0 \leq u<1$, by

$$
K\left(\left(e^{x}-1\right)<-u\right)=\int_{-1}^{-u} k_{X}(\ln (1+y)) \frac{1}{1+y} d y
$$

The right tail for gains $u>0$ is given by

$$
K\left(\left(e^{x}-1\right)>u\right) \int_{u}^{\infty} k_{X}(\ln (1+y)) \frac{1}{1+y} d y .
$$

The lower prudential valuation is then

$$
\begin{aligned}
& -\int_{0}^{1} \Lambda^{+}\left(\int_{-1}^{-u} k_{X}(\ln (1+y)) \frac{1}{1+y} d y\right) d u \\
& +\int_{0}^{\infty} \Lambda^{-}\left(\int_{u}^{\infty} k_{X}(\ln (1+y)) \frac{1}{1+y} d y\right) d u .
\end{aligned}
$$

Consider first the argument of $\Lambda^{-}$when the log arrival rates are of the variance gamma form with the $C G M$ parameterization. In this case the integrand for $\Lambda^{-}$is

$$
\begin{aligned}
C \int_{u}^{\infty} \frac{\exp (-M \ln (1+y))}{(1+y) \ln (1+y)} d y & =\int_{u}^{\infty} \frac{C}{(1+y)^{M}(1+y) \ln (1+y)} d y \\
& =\left.C \operatorname{Ei}(-M \log (1+y))\right|_{u} ^{\infty} \\
& =-C \operatorname{Ei}(-M \log (1+u)) \\
& =C \operatorname{expint}(M \log (1+u))
\end{aligned}
$$

For the integrand of $\Lambda^{+}$we have

$$
\begin{aligned}
\int_{-1}^{-u} k_{X}(\ln (1+y)) \frac{1}{1+y} d y & =-\int_{-1}^{-u} \frac{C(1+y)^{G}}{(1+y) \ln (1+y)} d y \\
& =\left.C \operatorname{Ei}(G \log (1+y))\right|_{-1} ^{-u} \\
& =-C \operatorname{Ei}(G \log (1-u)) \\
& =C \operatorname{expint}(-G \log (1-u))
\end{aligned}
$$

Hence the lower prudential valuation is

$V_{L}=-\int_{0}^{1} \Lambda^{+}(C \operatorname{expint}(-G \log (1-u))) d u+\int_{0}^{\infty} \Lambda^{-}(C \operatorname{expint}(M \log (1+u))) d u$ Making the change of variable $w=(1-u)$ in the first integral and $w=(1+u)$ in the second we obtain that 


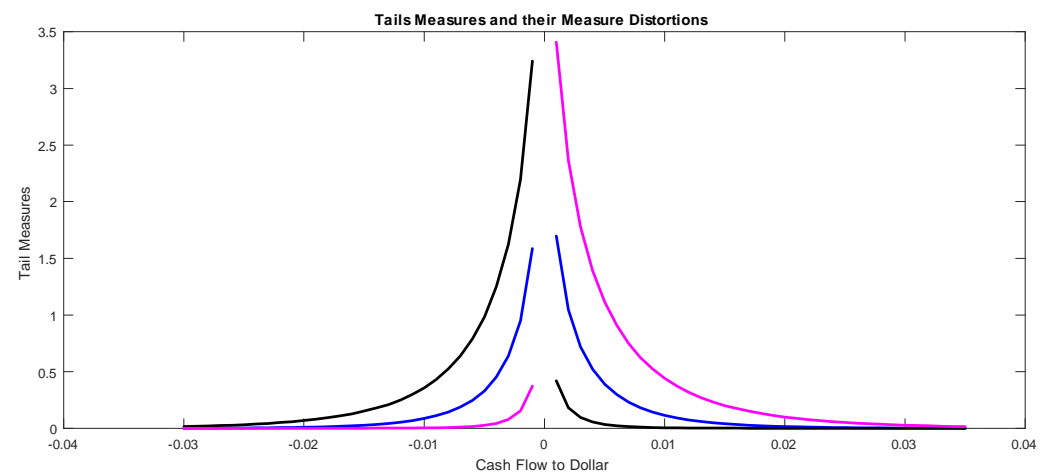

Figure 2: Tail Measures and their distortions. The tail measures are shown in blue. The lower distortion is in black and the upper distortion is in magenta.

$$
V_{L}=-\int_{0}^{1} \Lambda^{+}\left(C \operatorname{expint}(-G \log (w)) d w+\int_{1}^{\infty} \Lambda^{-}(C \operatorname{expint}(M \log (w))) d w\right.
$$

A similar analysis shows that the upper prudential valuation is

$$
V_{U}=\int_{1}^{\infty} \Lambda^{+}(C \operatorname{expint}(M \log (w))) d w-\int_{0}^{1} \Lambda^{-}(C \operatorname{expint}(-G \log (w))) d w
$$

\subsection{Illustrative Lower and Upper Prudential Variations based on Measure Distortions}

For the S\&P 500 index for September 28, 2015 reported on in Section 5 the $C$, $G$ and $M$ parameterization is respectively $1.1693,170.0209$ and 152.4098 . For the measure distortion parameters $a, b, c$ and $\gamma$ respectively at $3.1191,0.9694$, 0.3108 and 0.4877 . Figure 2 shows the tails measures and the distorted tail measures whose integrals appropriately differenced define the variation and the prudent lower and upper variations.

The lower and upper prudent variations were in basis points -153.43 and 176.71 while the exponential variation was 8.40.

\section{Calibration of Probability and Measure Dis- tortions}

In markets with prices continuously fluctuating the risk concern of markets may well be more with assessing how far up and down the prices may go and less with why at the moment it is any particular value. The latter leads us to covariation 
or beta type considerations while the former requires an assessment of safer or prudent upper and lower valuations and or variations. Clearly extreme distortions will give us absurdly wide limits with little empirical value while small distortions are likely to be equally useless providing zero risk characteristics in the limit.

The distortion stress parameters could be set at some intermediate level and left frozen at such levels but as markets get more nervous these levels may get progressively redundant or irrelevant. The same occurs as markets get less nervous and are willing to take on more risk. Ideally one would like to construct market calibrated distortion parameters that are progressively being adapted to current market conditions. There is then some hope that the spread and downside risk characteristics being computed are possibly empirically relevant.

An avenue pursued here is to calibrate to data from option markets and for an economy wide assessment of risk apetite we consider options on the S\&P 500 index. The discussion takes up probability distortions first and is generalized to measure distortions later. The lower and upper prudent valuations based on the distortion of physical probabilities has been seen to be an infimum and supremum of test probabilities $J$ performing test expectations. We understand that forward option prices are expectations of payoffs under a change of probability to a risk neutral probability often denoted $Q$. If the risk neutral probability $Q$ were to be among the collection of test probabilities defining the lower and upper valuations then option prices would be in the interval defined by the lower and upper valuation for the requisite cash flow. We also trust that with a small distortion is is unlikely that the option price is in the interval defined by the lower and upper valuations as both are then close to the physically expected cash and the option price is at some distance from such a value. Hence we seek the smallest distortion permitting risk neutral valuations to lie between the lower and upper prudential valuations.

The risk neutral distribution on the $\mathrm{S} \& \mathrm{P} 500$ index may be identified from option data on any day for any traded option maturity. We consider here the traded maturity closest to one month for the S\&P 500 index on any particular date. Denote by $F_{N}(x)$ this risk neutral distribution function as a function of $x=\ln (S(t+H) / S(t))$ for some horizon $H$ in days close to one month. Let $F_{P}(x)$ be the corresponding physical distribution function. The condition for the risk neutral probability to be among the test probabilities is that

$$
\widehat{\Psi}^{(\gamma)}\left(F_{P}(x, H)\right) \leq F_{N}(x) \leq \Psi^{(\gamma)}\left(F_{P}(x, H)\right) .
$$

and equivalently that the complementary probability $G_{N}(x)$ satisfy

$$
\begin{aligned}
1-\Psi^{(\gamma)}\left(F_{P}(x, H)\right) & \leq G_{N}(x) \leq 1-\widehat{\Psi}^{(\gamma)}\left(F_{P}(x, H)\right) \\
1-\Psi^{(\gamma)}\left(1-G_{P}(x, H)\right) & \leq G_{N}(x) \leq 1-\widehat{\Psi}^{(\gamma)}\left(1-G_{P}(x, H)\right) \\
\widehat{\Psi}^{(\gamma)}\left(G_{P}(x, H)\right) & \leq G_{N}(x) \leq \Psi^{(\gamma)}\left(G_{P}(x, H)\right) .
\end{aligned}
$$

The second inequality in (11) and the first in $(12)$ enforce risk neutral prices to be above the lower prudential valuations while the first in (11) and the second in 
(12) force risk neutral prices to be below the upper prudential vauations. Hence on the left for $x$ below the logartihm of the forward price relative to the spot we seek the second domination in (11) and on the right for $x$ above this critical value we seek the first domination in (12). The stress level is then sufficient to make market prices lie above the lower valuations. They may not lie below the upper valuations and one may need to raise stress levels further to attain the second domination. In the interests of keeping stress levels down we did not enforce the second set of inequalities. We seek the smallest $\gamma$ accomplishing the second inequality in (11) and equivalently the first inequality of (12).

This calibration exercise can be accomplished once we have the ability to evaluate $F_{P}(x, H)$. From time series data on daily returns we may employ our strategy of matching tail probabilities presented in Section 3 to estimate $F_{P}(x, h)$ where $h$ is the short horizon of one day. We next need a strategy for constructing long horizon $H$ distribution functions from short $h$ distribution functions.

Our law for one day returns is a self decomposable and hence infinitely divisible law that may be run as a Lévy process to the horizon $H$. However such a strategy suffers from a very fast decline in skewness and excess kurtosis that fall like the reciprocal of $\sqrt{H}$ and $H$ respectively. Eberlein and Madan (2010) observed that the data did not generally support such a rapid decline in the higher moments.

An alternative is to invoke self similarity considerations and scale the distribution by for example $\sqrt{H / h}$. Such a strategy has skewness and excess kurtosis not falling at all and one may observe from data that they do fall but just not as fast a Lévy process. Eberlein and Madan (2010) proposed combining the two methods by relying on the self decomposability of the law at the short horizon. Let $X_{h}$ be the self decomposable random variable at the short horizon. By self decomposability for every constant $\kappa, 0<\kappa<1$ there exists an independent random variable $X_{h}^{(\kappa)}$ such that

$$
X_{h} \stackrel{(d)}{=} \kappa X_{h}+X_{h}^{(\kappa)}
$$

The long horizon return suggested in Eberlein and Madan (2010) is to define $X_{H}$ as the sum of running $\left(\kappa X_{h}\right)$ as a Lévy process to time $H$ and scaling the independent component $X_{h}^{(\kappa)}$. Hence we propose to take

$$
X_{H} \stackrel{(d)}{=}\left(\kappa X_{h}\right)_{H}+\left(\frac{H}{h}\right)^{\eta} X_{h}^{(\kappa)} .
$$

One may simulate from $X_{H}$ directly as the law of $X_{h}^{(\kappa)}$ may be identified and simulated from. Skewness and excess kurtosis fall with $H$ for $X_{H}$ but at rate slower than that observed for Lévy processes. The random variable $X_{H}$ is not in the variance gamma class but we approximate it by a variance gamma law by fitting a variance gamma model to the simulated data. This procedure gives us $F_{P}(x, H)$ in the variance gamma class. We follow Eberlein and Madan (2010) by employing the suggested values for $\kappa=0.5$ and $\eta=0.5$. 


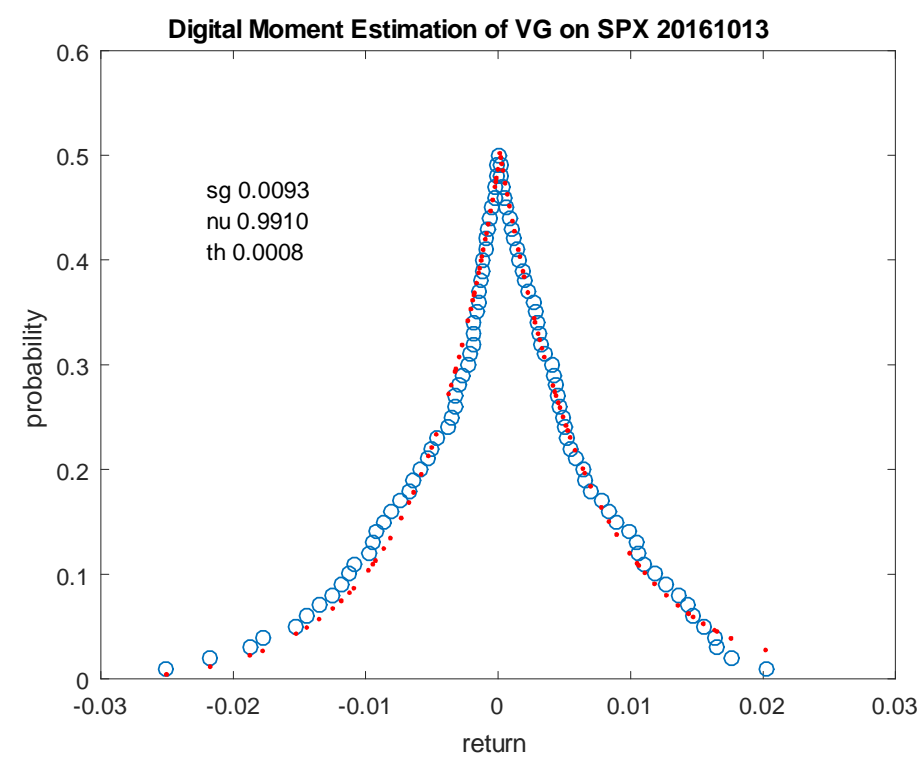

Figure 3: The observed tail probabilities are presented as circles. The model probabilities are represented by dots. Also shown are the parameter estimates.

By estimating the risk neutral distribution at the monthly maturity in the variance gamma class we obtain a risk neutral arrival rate function $k_{N}(x)$. A variance gamma approximation to the long horizon return delivers a physical arrival function $k_{P}(x)$. For the calibration of measure distortions the conditions mirror those for probability distortions and require that for all $a>0$

$$
\begin{gathered}
\int_{-\infty}^{-a} k_{N}(x) d x \leq \Gamma^{+}\left(\int_{-\infty}^{-a} k_{P}(x) d x\right) \\
\int_{a}^{\infty} k_{N}(x) d x \geq \Gamma^{-}\left(\int_{a}^{\infty} k_{P}(x) d x\right) .
\end{gathered}
$$

The parameters for the measure distortion are selected each day to meet these conditions via a minimal distortion.

\subsection{Sample calibration of a probability distortion}

For the S\&P 500 index on October 13, 2016 using 252 days of prior continuously compounded daily return data we estimate by matching digital moments the variance gamma model. Figure 3 presents the observed and fitted tail probabilities.

The number of days to the option maturity in this case was 30 days. The long horizon 30 day returns were generated by shaving the daily variance gamma law by a half running this as an i.i.d. process for 30 days and adding to this 


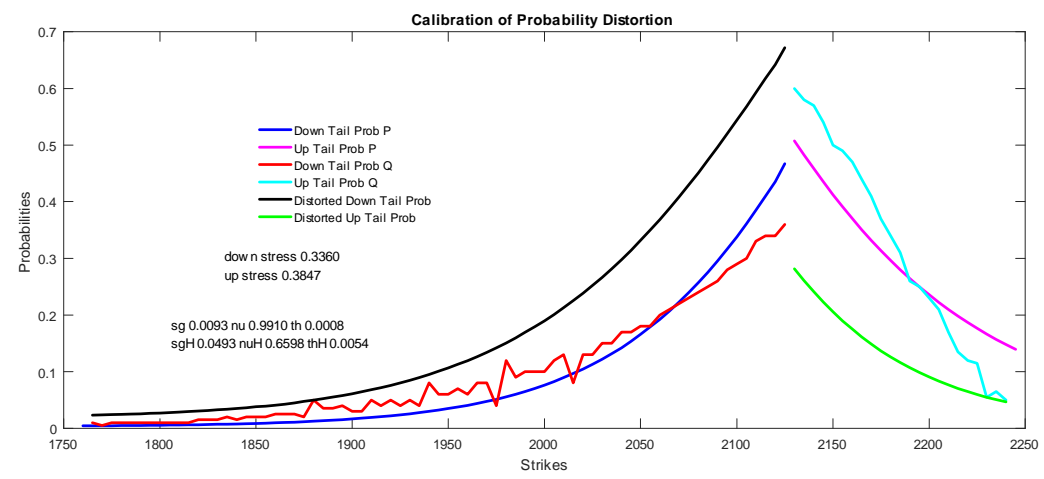

Figure 4: Physical, risk neutral and distorted physical donw and up side tail probabilities.

the independent component scale by $\sqrt{30}$. The variance gamma approximation to the long horizon return had the parameters

$$
\sigma=0.0493 ; \nu=0.6598 ; \theta=0.0054 .
$$

These parameters were used to evaluate down and up side physical tail probabilities. The corresponding risk neutral probabilities were obtained directly from put and call prices by evaluating the derivatives of call and put prices. Distorted down probabilities were constructed to attain domination from above of the risk neutral probabilities. Distorted up probabilities were constructed to attain domination from below of risk neutral probabilities. Figure 4 presents all probabilities and the stress parameters required on each side. The stress level for the day is the larger of the two. The two stress levels were 0.3360 and 0.3847 .

\subsection{Sample calibration of a measure distortion}

For the calibration of a measure distortion we begin by identifying the risk neutral tail measure. The physical tail measure is as it was identified for the probability distortion. For this purpose we fit a risk neutral model to options prices across strikes and maturities as we may then identify parameters for arbitrary maturities as opposed to just exchange traded maturities. Carr, Geman, Madan and Yor (2007) formulated the Sato process base on the variance gamma at unit time that has a variance gamma risk neutral law at all maturities. In addition to the three variance gamma parameters at unit time there is an additional scaling parameter $\rho$ as the density at time $t$ is in distribution that of $t^{\rho} X$ where $X$ is the unit time variance gamma law. Sato (1991) showed that every self decomposable law at unit time when scaled to the other maturities is consistent with an additive process of independent but inhomogeneous increments that have the scaled densities as their marginals. Carr, Geman, Madan and Yor (2007) termed this process when the unit time law is variance gamma the 


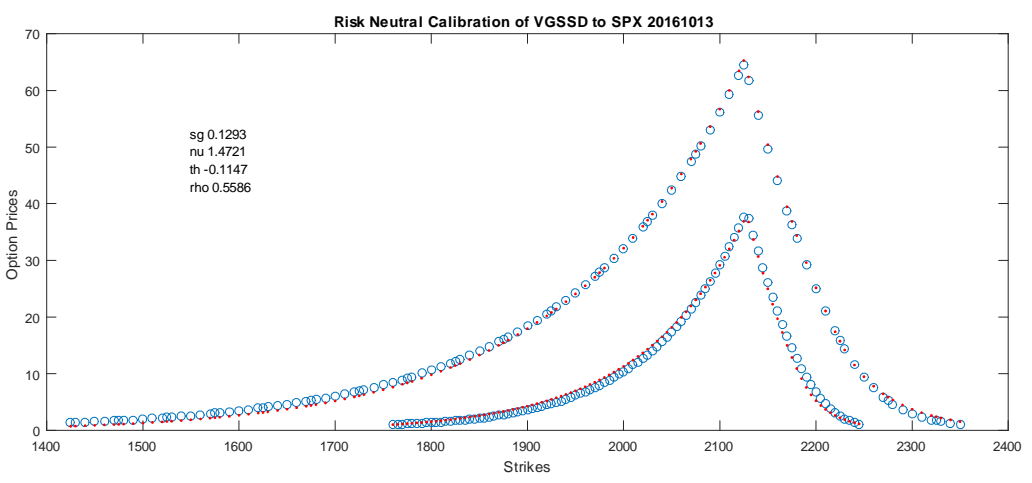

Figure 5: Fit of VGSSD to option prices on S\&P 500 index as at October 13, 2016. Market prices are represented by blue circles while model prices are shown by red dots.

$V G S S D$ process for variance gamma, scaled self decomposable. For the option data on October 13, 2016 on the S\&P 500 index we fit the VGSSD model for two maturities close to one and three months. Figure 5 presents the fit of the model to observed option prices along with the Sato process parameters.

From this fit one may identify a risk neutral tail measure in the variance gamma class for the one month maturity. The corresponding physical tail measure in the variance class was already identified from the long horizon approximation introduced earlier. For the distortions $\Lambda^{+}, \Lambda^{-}$we need to identify four parameters, $a, b, c, \gamma$. By symmetry we set $a=b / c$. We define $b$ by recognizing that $\Lambda^{-\prime}(0)=1-b$. Equating $\Lambda^{-\prime}(0)$ to the derivative of the risk neutral measure at zero identifies $b$. The parameter $c$ is selected to accomplish the required domination on the right while the parameter $\gamma$ is selected to attain the domination on the left. Figure 6 presents the physical, risk neutral and distorted physical tail measures on both sides along with the measure distortion parameters.

\section{Results on Required Returns}

The empirical results are presented in subsections.

\subsection{Required Returns and Risk Characteristics}

The probability and measure distortions were calibrated to data on S\&P 500 index options from January 4, 2010 to March 3, 2016. For each of 1552 days in this time period for all of 229 stocks, 9 sector ETF's and the S\&P 500 index or a total of 239 underlying assets the lower and upper prudential valuations were computed. The computations were based on the empirical tail probabilities, the fitted variance gamma tail probabilities and the measure distorted fitted variance gamma arrival rate functions. In each of these three cases two risk 


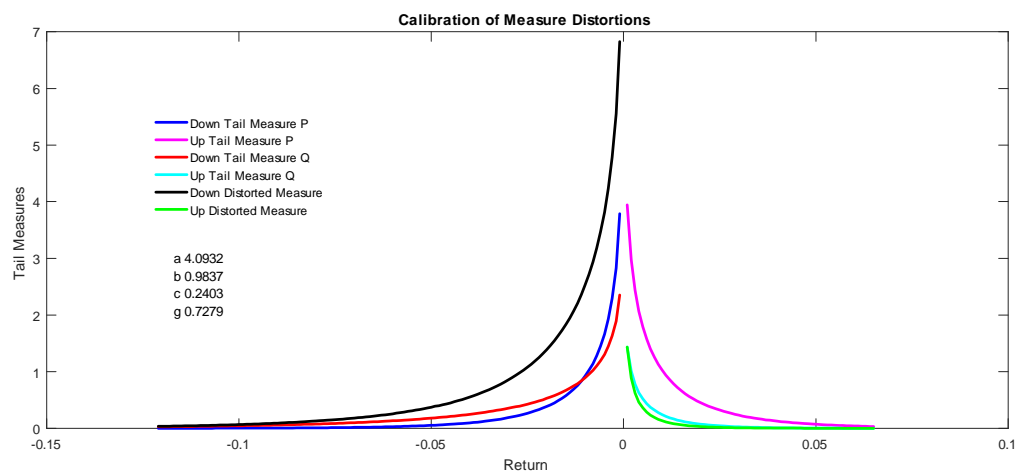

Figure 6: Physical, risk neutral and distorted physical tail measures for the S\&P 500 index on October 13, 2016.

characteristics were constructed. The first is a spread or capital charge measure defined as

$$
s_{i t}=\ln \left(\frac{1+U_{i t}}{1+L_{i t}}\right) .
$$

The second is downside risk measure defined as

$$
d_{i t}=-\ln \left(1+L_{i t}\right) .
$$

Each of the two risk characteristics is based on 252 days of immediately past daily continuously returns addressing the risk in the cash flow $e^{x}-1$ where $x$ is the daily $\log$ price relative for the calculations based on daily data or the variance gamma probability model for the daily log price relative and $x$ is just the jumps size in the case of the arrival rate function and the measure distortions.

A cross sectional regression was run each day across the 239 underlying assets where the dependent variable was the mean daily percentage change over the last 252 days for the case of the raw data with no probability model. For the variance gamma model fitted by matching tail probabilities the dependent variable was the expected return as per equation (3). For the measure distortions the dependent variable was the exponential variation of equation (8). The independent variables were in addition to a constant, the spread and downside risk characteristics. These are computed in the three cases by distortion of the empirical tail probabilities, the model tail probabilities, and finally measure distortion of the arrival rate functions. There are three cross sectional regressions for each of 1552 days. The predictions from the regressions constitute three sets of required returns with a minimum required return set a half a basis point. The ratios of the dependent variable to the required returns define the value of the invested dollar.

Figure 7 presents the three sets of $R^{2 \prime} s$ for the 1552 days. We observe that the best performance is offered by the use of measure distortions, while the 


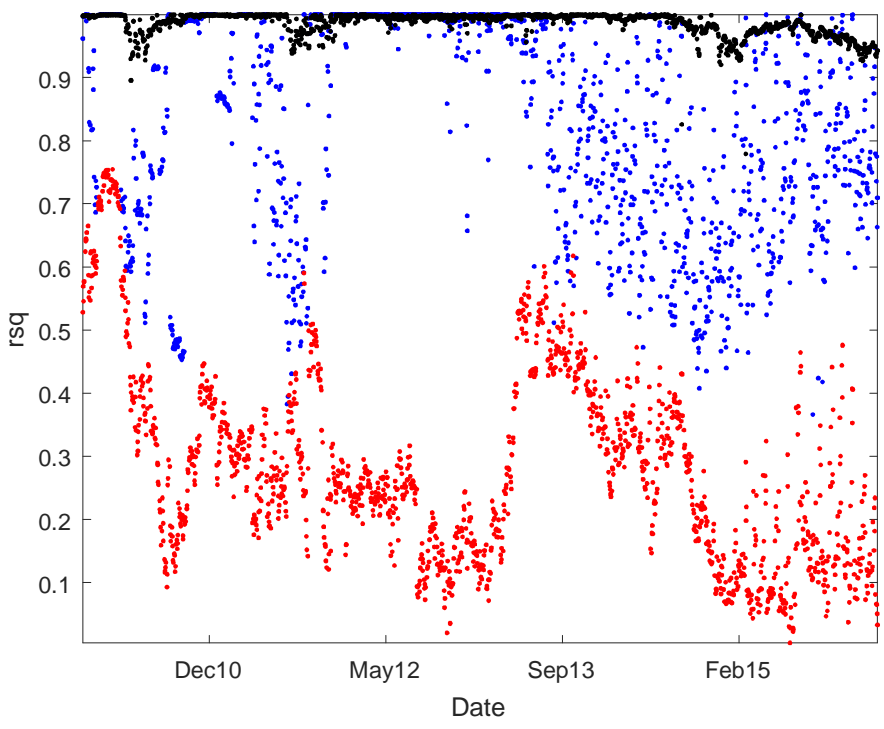

Figure 7: The RSQ for the empirical tail probability distortion are presented in blue. Those for the distortion of variance gamma model probabilities are in red and the measure distortion is in black.

distortion of empirical tail probabilities gives a better result than the distortion of model tail probabilities.

Figure 8 presents a graph of the coefficient for the capital risk characteristic using the three regressions. The results from the use of measure distortions are the most stable and close to a half for a fair while. The results from the probability distortions are quite volatile and the empirical tail probability distortions are also volatile but less so.

Figure 9 presents the coefficients for the characteristic on downside risk exposure. The probability distortion coefficient is quite volatile and is presented separately in Figure 10 The empirical tail probability often has a negative coefficient possibly due to the limited reach of data sets. The measure distortion is quite stable around the $2 \%$ mark.

By way of the t-statistics Table 1 presents the quartiles for the three sets of 


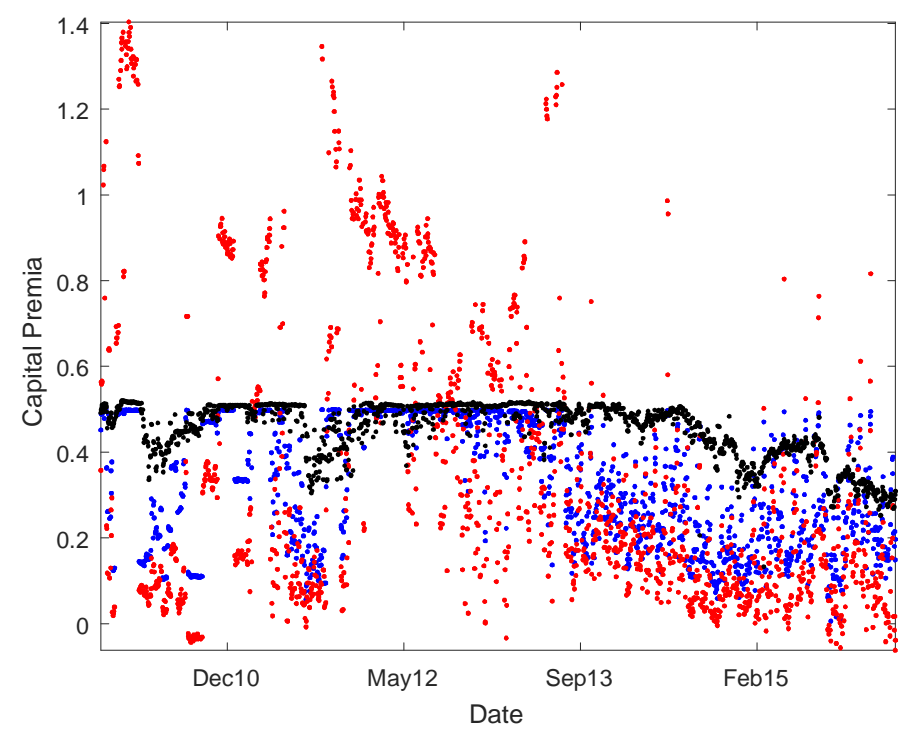

Figure 8: Capital charge coefficients. Empirical tail probability distortions are in blue, probability distortions in red and measure distortions in black.

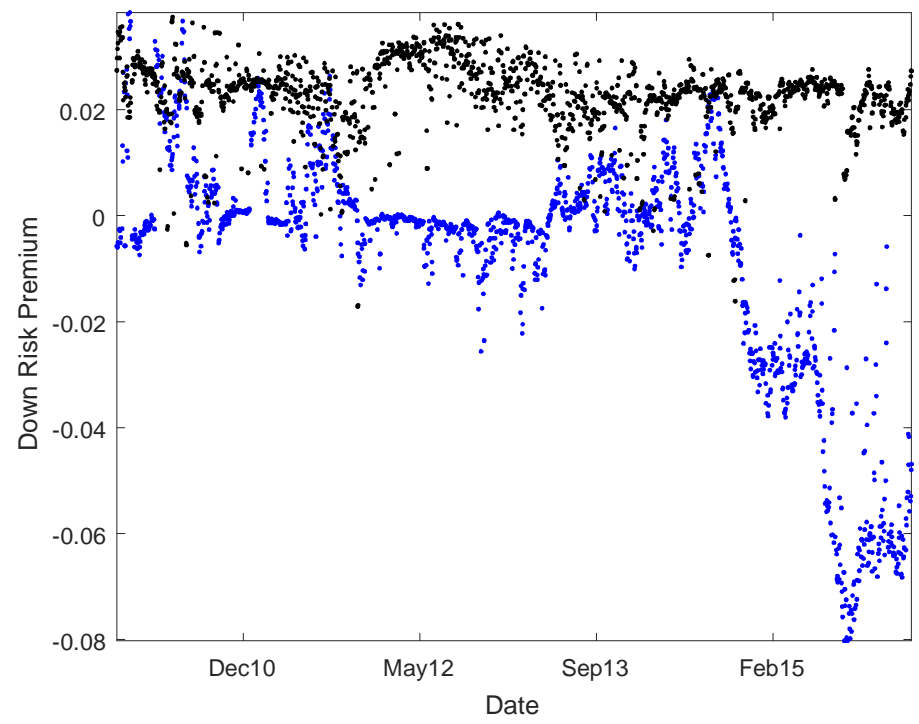

Figure 9: Down Risk Premia. Empirical tail distortion in blue and measure distortion in black. 


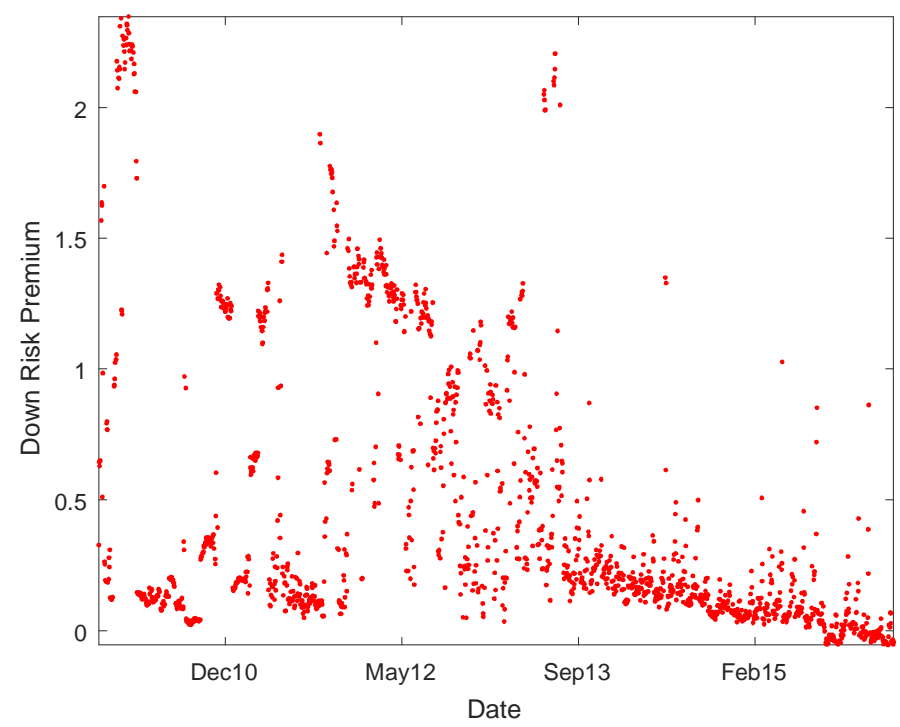

Figure 10: Down Risk Premium coefficients for probability distortions.

t-statistics on the two coefficients for the capital and downrisk characteristics.

\section{TABLE 1}

quartiles

$\begin{array}{lllll} & & 1 & 2 & 3 \\ \text { Raw } & \text { Capital } & 19.83 & 38.15 & 362.4 \\ & \text { DownRisk } & -3.0 & -0.76 & 0.90 \\ \text { Probability } & \text { Capital } & 3.64 & 5.90 & 8.69 \\ \text { Model } & \text { DownRisk } & 5.72 & 8.54 & 10.95 \\ & & & & \\ \text { Measure } & \text { Capital } & 100.79 & 208.75 & 371.85 \\ \text { Distortion } & \text { DownRisk } & 19.74 & 34.56 & 52.61\end{array}$

The down risk is not significant in the empirical tail distortion. Both premia are significant for the probability distortion, but as already observed the coefficients are quite variable. The measure distortion yields highly significant premia for both risks.

\subsection{Required Returns based on Tail Risk and Beta}

The results for the $R^{2}$ were similar to those without the addition of the asset beta. The capital and down risk premia were also similar. Table 2 presents the quartiles for the t-statistics on the three coefficients. With the raw data beta 
is generally positive but not significant. With the probability distortion and measure distortion when significant it has the wrong sign. We conclude this section by observing that the use of beta in asset pricing may not be called for once risk characteristics have been introduced.

\begin{tabular}{lllll}
\multicolumn{5}{c}{ TABLE 2} \\
quartiles \\
Raw & Capital & 1 & 2 & 3 \\
Data & DownRisk & -3.74 & 33.22 & 242.3 \\
& Beta & 0.42 & 1.65 & -0.93 \\
& & & & \\
Probability & Capital & 2.84 & 5.35 & 8.12 \\
Model & DownRisk & 4.68 & 6.23 & 7.73 \\
& Beta & -3.12 & -1.83 & 0.52 \\
Measure & Capital & 100.4 & 207.2 & 352.6 \\
Distortion & DownRisk & 14.39 & 26.26 & 36.97 \\
& Beta & -4.11 & -2.62 & -2.14
\end{tabular}

\subsection{Relative Contributions of Capital Charges and Down- side Risk Components of Required Returns}

For each of the 1552 days and 239 assets we evaluated the share of the required return made up by the contribution of the capital charge. This is the variable $s_{i t}$ times its coefficient divided by the sum of this component and variable $d_{i t}$ times its coefficient. The proportion of positive required returns was $94.34,45.19$ and 84.90 percent respectively for the measure distortion applied to the arrival rate function, the probability distortion applied to the model probability and the probability distortion applied to the empirical distribution. In these positive cases we evaluated the quartiles for share of the capital charges in the required return.

For the measure distortion the quartiles were $67.13,78.39$ and 86.01 respectively. The corresponding values for the probability distortion applied to the probability model were $7.48,18.19$ and 43.09 . The use of the empirical distribution had proportions of 85.40 and 100 percent.

We may conclude that the use of the empirical distribution is curtailed in its ability to address downside risk by the limited structure of outcomes it is working with. The probability distortion applied to the probability model has considerable weight on downside risk but the results have to be tempered by the numerical approximations involved. The measure distortion delivers a more reasonable and stable set of results. 


\section{Results on the Value of an Invested Dollar}

Results for the traditional alpha beta approach is followed by the presentation of a risk characteristics apporach in subsections of this Section.

\subsection{Alpha and Beta Adjusted Alpha Rankings}

For the time period January 3, 2008 to November 17, 2016 for a period covering 2237 days the alphas $\alpha_{i t}$, betas $\beta_{i t}$ and value of an invested dollar $v_{i t}$, defined as

$$
v_{i t}=1+\frac{\alpha_{i t}}{\beta_{i t}},
$$

were evaluated. The index $i$ covered 229 stocks and 9 sector exchange traded funds or a total of 238 assets. The index $t$ runs through the time period of 2237 days. The computations each day were based on a year's set of immediately past daily returns. A time series regression of asset returns for the prior year on a constant and the contemporaneous return on the $S \& P 500$ index return delivered the asset beta as the coefficient on the index return. A cross-sectional regression each day of daily returns averaged over the prior year on a constant and the asset beta yields the market risk premium as the coefficient for the beta. The alpha is constructed as the average daily return over the year less the asset beta times the market risk premium.

Figures 11 and 12 present the marginal distributions for the alpha and the value of an invested dollar across the 532, 406 asset days.

The marginal distributions appear comparable but the null hypothesis of identical marginal distributions for the data on alpha and the alpha beta ratio is rejected by a Kolmogorov Smirnov test at the $5 \%$ level. The test statistic was 0.0099 .

Similar tests may be conducted for identity of distributions across stocks for each day and identity of distributions across time for each stock. For the former the null hypothesis of an identical distribution across stocks each day is not rejected but the hypothesis of an identical distribution across time for each stock is rejected for 87.82 percent of the stocks. If one tests for an identity of distributions across asset days for all assets across three weeks or 15 days at a time then this identity is rejected $48 \%$ of the time.

Figure 13 displays a sample of cases where $\alpha$ is high and the value of the invested dollar as measured by the alpha beta ratio is low and vice versa. In conclusion, the alpha beta ratio can differ from the alpha as a measure performance.

With regard to the value of invested dollar Figures 14, 15 and 16 present the probability distribution of this value across the 370,928 asset days using the raw data, the probability model and the measure distorted arrival rate functions. The most stable results are with the measure distorted arrival rate functions followed by the probability model and the then the raw data that has a long negative tail which was truncated for presentation. 


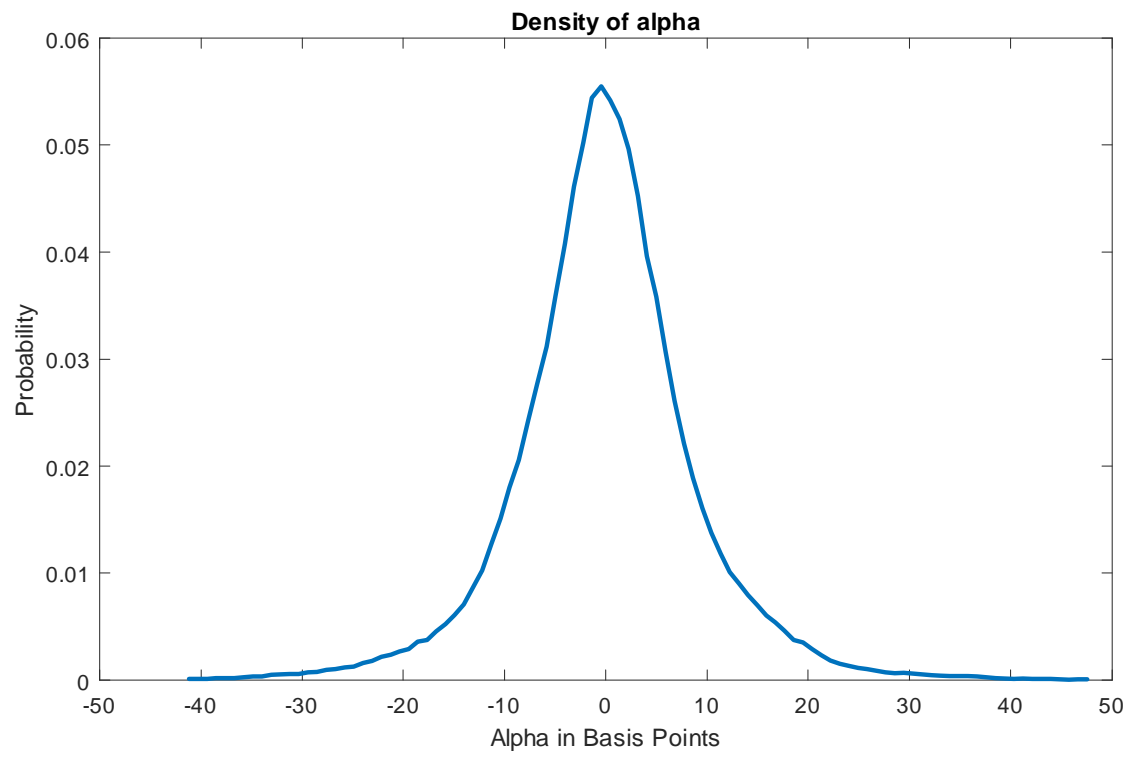

Figure 11: Density of alpha in basis points.

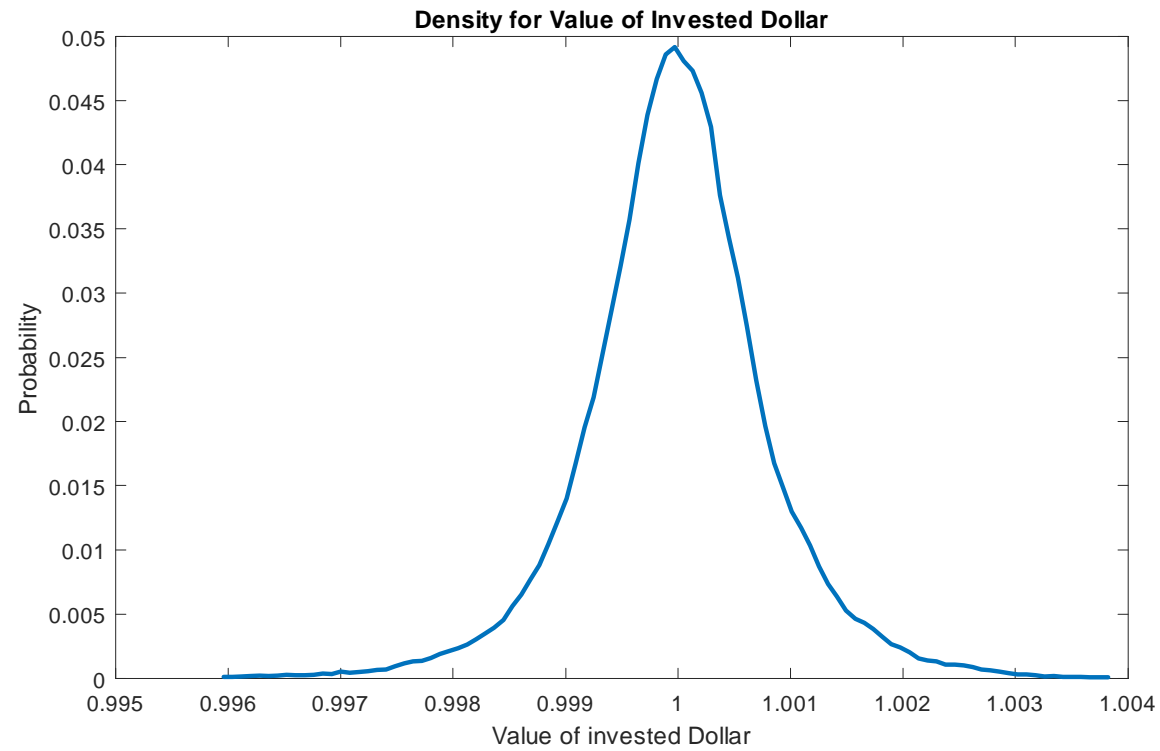

Figure 12: Density of Value of Invested Dollar based on an S\&P 500 index beta model for required returns. 


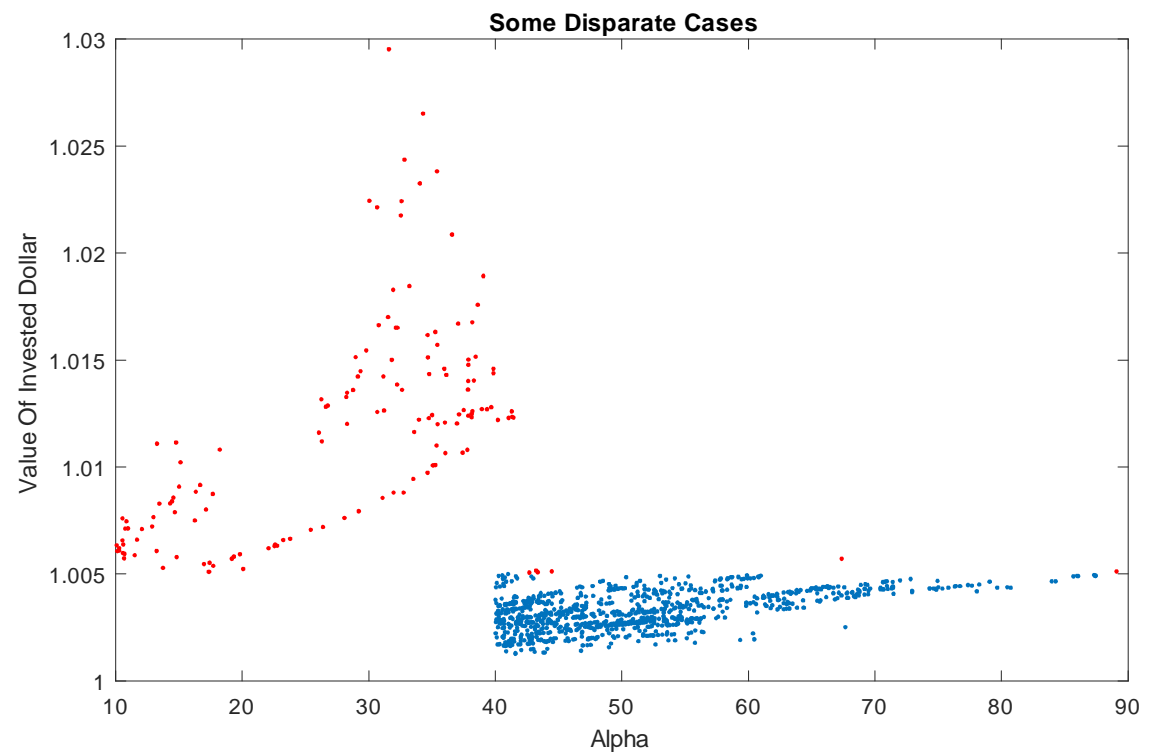

Figure 13: Sample of cases with high $\alpha$ and low value of invested dollar and vice versa.

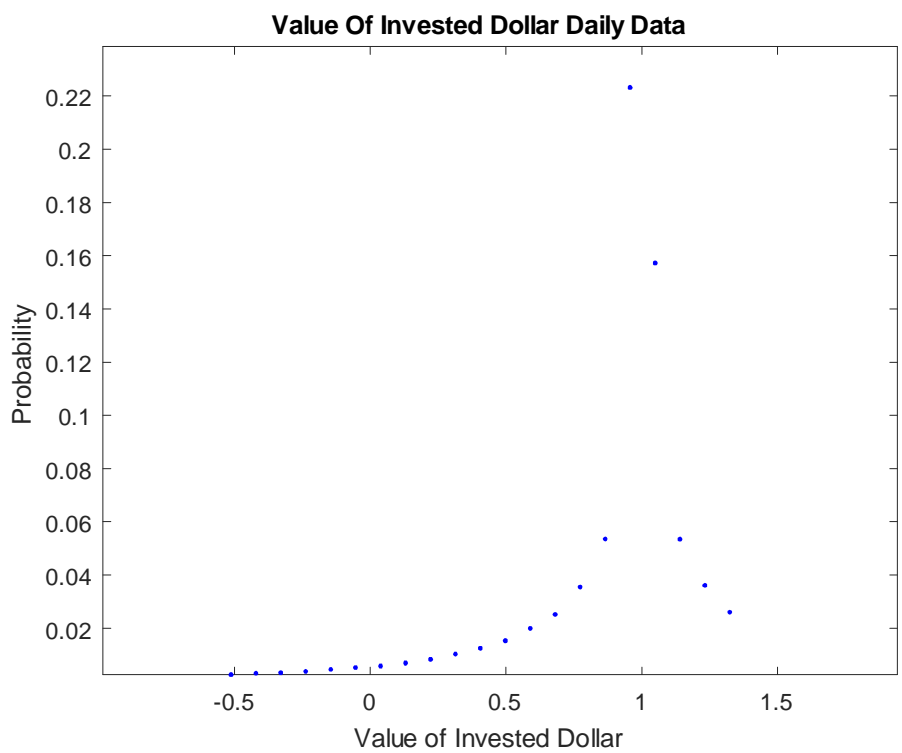

Figure 14: Distribution of the value of an invested dollar using the raw data for daily returns and no distributional model. 


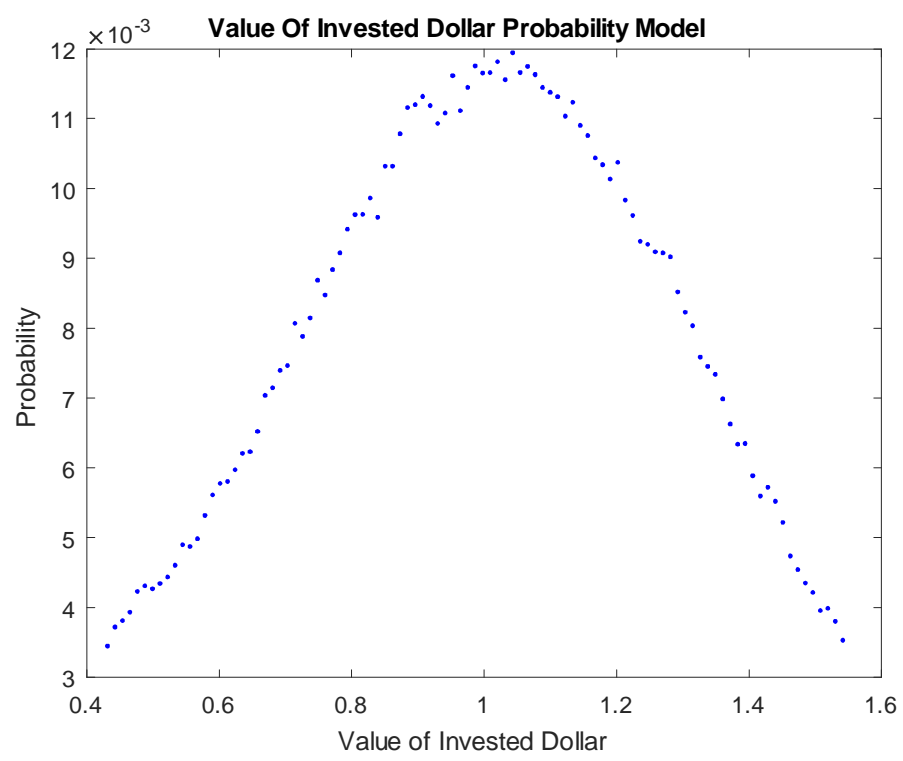

Figure 15: Distribution of the value of an invested dollar using the variance gamma probability model and its distortion.

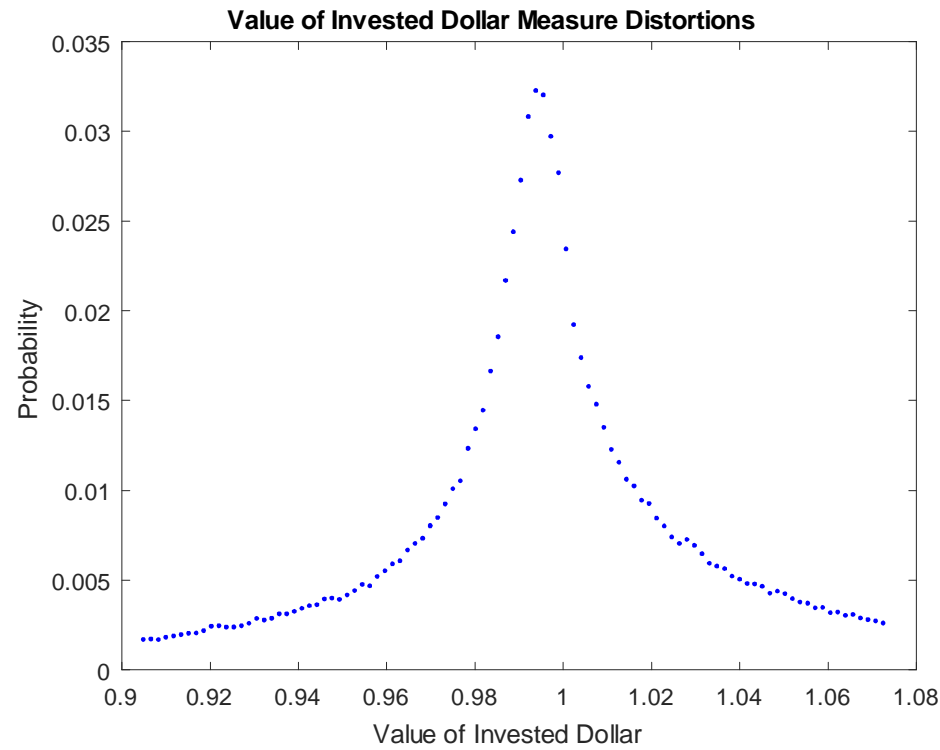

Figure 16: Distribution of the value of an invested dollar using a variance gamma arrival rate function that is measure distorted for the risk characteristics. 


\subsection{Asset Rankings based on Risk Characteristics}

Each day the assets may be ranked on the basis of alpha or the value of invested dollar as evaluated by the alpha beta ratio, the measure distortion, probability distortion or the distortion of the empirical distribution function. These rankings may be termed, $a, a b, m, p$ and $e$ for the five ways in which they may be generated. Table 3 presents the quartiles across the 1552 days for the correlations between these five rankings. Apart from alpha and the alpha beta ratio the value of the invested dollar evaluated via measure distortions, probability distortions or the empirical distribution deliver different rankings.

TABLE 3

\begin{tabular}{cccc}
\multicolumn{4}{c}{ Ranking Correlations } \\
& 1 & 2 & Quartiles \\
a,ab & 0.9770 & 0.9822 & 0.9877 \\
$\mathrm{a}, \mathrm{m}$ & 0.1175 & 0.1917 & 0.2829 \\
$\mathrm{a}, \mathrm{p}$ & 0.3352 & 0.4241 & 0.5167 \\
$\mathrm{a}, \mathrm{e}$ & 0.4689 & 0.5963 & 0.7449 \\
$\mathrm{ab}, \mathrm{m}$ & 0.0852 & 0.1709 & 0.2692 \\
$\mathrm{ab}, \mathrm{p}$ & 0.3295 & 0.4190 & 0.5154 \\
$\mathrm{ab}, \mathrm{e}$ & 0.4805 & 0.5913 & 0.7116 \\
$\mathrm{~m}, \mathrm{p}$ & 0.2629 & 0.3242 & 0.3797 \\
$\mathrm{~m}, \mathrm{e}$ & 0.1917 & 0.2796 & 0.3629 \\
$\mathrm{p}, \mathrm{e}$ & 0.4429 & 0.5581 & 0.6793 \\
& & &
\end{tabular}

\section{Conclusion}

Risk characteristics based on prudent upper and lower valuations as they appear in abstract models of arbitrage free two price economies are introduced as explanatory variables to be compensated for in forming required returns. Asset prices are seen to be of necessity in continuous motion. The risks of positions in financial markets are then about how far up and down they may safely be viewed to fluctuate. The pricing principles of two price economies suggest ways of calibrating such price spreads and the associated depth of down movements. Two risk characteristics based on the gap between the upper and lower price and the level of the lower price are seen to provide an adequate explanation of expected returns. They are then used to formulate required returns. The value of the invested dollar is modeled as the ratio of the expected return to the required return.

The spread and downside market depth is modeled using parametric models for probability distortions at the level of daily data. In the continuous time limit one applies parameteric measure distortions to the tail measures of jump arrival rates. Both the probability and measure distortions are calibrated to the market for options on the S\&P 500 index. It is observed that frozen distortion parameters can lead to drifts in the value of the invested dollar away from unity 
probably due to obsolete or unrealistic distortion stress levels.

The results show a better performance delivered on employing the analytical structure of measure distorting jump arrival rates. The two risk characteristics are both highly significant in explaining expected returns especially when applying measure distortions. There is also little room or reason for the inclusion of betas or possibly other covariation based measures once risk characteristics have been introduced. The relative contribution of capital charges to required returns has an interquartile range of 67 to 86 percent when assessed by measure distortions applied to arrival rate functions. The different constructions for the value of an invested dollar also deliver different asset rankings across time. 


\section{References}

[1] Back, K. E. (2010), Asset Pricing and Portfolio Choice Theory, Oxford University Press, Oxford, U.K.

[2] Barndorff-Nielsen, O. E. and A. Shiryaev (2010), Change of Time and Change of Measure, World Scientific Press, Singapore.

[3] Bion-Nadal, J. (2009), "Bid-Ask Dynamic Pricing in Financial Markets with Transactions Costs and Liquidity Risk," Journal of Mathematical Economics 45, 738-750.

[4] Black, F. (1993), "Estimating Expected Return," Financial Analysts Journal, September/October 36-38.

[5] Brennan, M. J., T. Chordia and A. Subrahmanyam (1998), "Alternative factor specifications, security characteristics, and the cross-section of expected stock returns," Journal of Financial Economics, 49, 345-373.

[6] Carr, P., H. Geman, D. B. Madan and M. Yor (2007),"Self-Decomposability and Option Pricing," Mathematical Finance, 17, 31-57.

[7] Carr, P., D. B. Madan, and J. J. Vicente Alvarez (2011), "Markets, Profits, Capital, Leverage and Returns," Journal of Risk 14, 95-122.

[8] Cherny, A., Madan, D. B.: New Measures for Performance Evaluation. Review of Financial Studies 22, 2571-2606 (2009)

[9] Choquet, G.: Theory of Capacities. Annales de l'Institut Fourier 5, 131-295 (1953).

[10] Chordia, T., A. Goyal and J. Shanken (2015), "Cross-Sectional Asset Pricing with Individual Stocks: Betas versus Characteristics," Working Paper, Emory University, available at https://ssrn. com/abstract $=2549578$

[11] Eberlein, E. and D. B. Madan (2010), "The Distribution of Returns at Longer Horizons," Recent Advances in Financial Engineering; Proceedings of the KIER-TMU workshop. Eds. M. Kijima, C. Hara, Y. Muromachi, H. Nakaoka and K. Nishide, World Scientific, Singapore

[12] Eberlein, E., D. Madan, M. Pistorius and M. Yor (2014), "Bid and Ask Prices as Non-Linear Continuous Time G-Expectations Based on Distortions," Mathematical and Financial Economics, 8, 265-289.

[13] Fama, E. F. and K. R. French (1992), "The Cross-Section of Expected Stock Returns," Journal of Finance, 47 427-465.

[14] Fama, E. F. and K. R. French (1993), "Common risk factors in the returns on stocks and bonds," Journal of Financial Economics, 33, 3-56. 
[15] Guasoni, P., E. Lepinette and M. Rasonyi (2012), "The Fundamental Theorem of Asset Pricing under Transactions Costs," Finance and Stochastics $16,741-777$.

[16] Jouini, E., and H. Kallal (1995),"Martingale and Arbitrage in Securities Markets with Transaction Cost," Journal of Economic Theory 66, 178-197.

[17] Khintchine, A. Y. (1938), "Limit laws of sums of independent random variables," ONTI, Moscow, (Russian).

[18] Lévy, P. (1937), "Théorie de l'Addition des Variables Aléatoires," GauthierVillars, Paris.

[19] Lintner, J. (1965), "The Valuation of Risk Assets and the Selection of Risky Investments in Stock Portfolios and Capital Budgets," Review of Economics and Statistics, 47, 13-37.

[20] Madan, D. B. (2015), "Estimating Parameteric Models of Probability Distributions," Methodology and Computing in Applied Probability, 17, 823831 .

[21] Madan, D. B. (2015a), "Asset Pricing Theory for Two Price Economies," Annals of Finance, 11, 1-35.

[22] Madan, D. B. (2016), "Instantaneous Portfolio Theory," Working Paper Robert H. Smith School of Business, available at https://ssrn.com/ abstract $=2804718$

[23] Madan, D. B. (2017), "Efficient Estimation of Expected Stock Price Returns," Working Paper Robert H. Smith School of Business, available at https://ssrn. com/abstract=2894499

[24] Madan, D., Carr, P. and Chang, E (1998), "The variance gamma process and option pricing," European Finance Review, 2, 79-105.

[25] Madan, D. B. and W. Schoutens (2016), Applied Conic Finance, Cambridge University Press, Cambridge, UK.

[26] Madan, D., and Seneta, E. (1990), "The variance gamma (VG) model for share market returns," Journal of Business, 63, 511-524.

[27] Mossin, J. (1966), "Equilibrium in a Capital Asset Market," Econometrica, 35, 768-783.

[28] Ross, S.A. (1976), "Arbitrage Theory of Capital Asset Pricing," Journal of Economic Theory, 13, 341-360.

[29] Ross, S. (1978), "A Simple Approach to the Valuation of Risky Streams," Journal of Business 51, 453-475. 
[30] Sato, K. (1991), "Self similar processes with independent increments," Probability Theory and Related Fields, 89, 285-300.

[31] Sato, K. (1999), Lévy processes and Infinitely Divisible Distributions, Cambridge University Press, Cambridge.

[32] Sharpe, W.F. (1964), "Capital Asset Prices: A theory of Market Equilibrium under Conditions of Risk," Journal of Finance, 19, 425-442. 